\title{
EXPERIENCES OF COAL FIRE DETECTION AND QUANTIFICATION FOR RESOURCES MANAGEMENT
}

\author{
Christian Fischer ${ }^{1}$, Stefan Schloemer ${ }^{2}$, Andreas Hirner ${ }^{1}$, Manfred Teschner ${ }^{2}$ \\ ${ }^{1}$ German Aerospace Center, German Remote Sensing Data Center, Germany \\ ${ }^{2}$ Federal Institute for Geosciences and Natural Resources, Germany
}

\begin{abstract}
Uncontrolled coal seam fires are an environmental and economic problem of international magnitude. Today, China is the leading country of coal production, consumption and export. The annual Chinese coal production, which is about approx. 2.4 Mt per year, faces a problem of numerous uncontrolled burning coal fires mostly located in the northern Chinese coal belt. The determination of fire radiative energy (FRE) has been introduced recently as a new remote sensing technique to quantify forest and grassland fires. The main research in this project focuses on the development of robust routines to detect coal fire related thermal anomalies within the different areas under investigation. These developments are the prerequisite for a coal fire monitoring.
\end{abstract}

Index Terms - thermal remote sensing, resources, gas and temperature measurements, energy release, CDM

\section{INTRODUCTION}

Comprehensive studies focuses on two different test sites located in the Autonomous Region of Inner Mongolia and in Xinjiang Uyghur Autonomous Region. Both areas under investigation are heavily affected by severe coal fires since years on different places within the coalfields. Besides subsurface coal fires, surface coal fires occur directly on outcropping seams and on places where small-scale mining took place. Thermal anomaly detection takes the sensor sensitivity, the size of the anomaly and the individual background temperature on different locations into account. Due to the different geological situations, different fire sizes and fire temperatures as well as the changing background pixel statistics, adaptive thresholds and adaptive temperature anomaly detection tests have been developed. This research aims to establish remote sensing analysis and the modeling techniques as an integral method, conjointly together with geo-physical measurement techniques and coal fire extinction activities, within the framework of the Kyoto Protocol.

\section{COAL FIRE DTECTION}

Recording the areas under investigations by ASTER night time images at regular times in 2007 were possible based on a proposal for scientific use of ASTER data accepted by the ASTER science team.

Different approaches exist to detect and to analyze vegetation fires. But coal fires do not only occur exposed and the following aspects have to be taken into account:

- coal fire temperatures are varying strongly,

- most fires are subsurface with a crack system at topographic surface,

- mostly surface temperatures below $600^{\circ} \mathrm{K}$,

- fire dynamic is unknown,

- $\quad$ energy release is based on radiated energy, emitted energy by conduction and conversion and transported by emitted gases.

So it is important to mention that all remote sensor systems can only register that part of the total fire energy that is released as radiation. In addition, a fire zone is of irregular geometrical shape normally, thus a pixel will contain both fire and background information. With respect to the Stefan-Boltzmann law, the amount of radiative energy release of a fire related sub-pixel can be described as follows [1]:

$$
M_{\text {fire }}=A_{\text {pixel }} \sigma \varepsilon \sum q_{f n} T_{f n}^{4}
$$

where:

$\mathrm{M}_{\text {fire }}$ : radiative energy release of the fire (FRE) [W]

$\mathrm{A}_{\text {pixel }}$ : ground sampling area $\left[\mathrm{m}^{2}\right]$

$\sigma \quad$ Stefan-Boltzmann constant [Wm² K-4]

$\varepsilon \quad$ emissivity

$\mathrm{q}_{\mathrm{fn}} \quad$ fractional areas of the thermal component

$\mathrm{T}_{\mathrm{fn}} \quad$ temperature of the thermal component 
The method to compute fire related energy directly from the spectral radiances recorded by the ASTER sensor system is based on the technique presented by [2]. It is based on the assumption the total energy release is linearly proportional to the pixel radiance recorded in the TIR spectral range. Theoretical simulations, taking the sensor sensitivity into account, have been made to calculate the sensor specific constants.

$$
M_{\text {fire }}=b A_{\text {pixel }}\left(L_{p}-L_{b g}\right)
$$

where:

$\mathrm{M}_{\text {fire }}$ : radiative energy release of the coal fire

b: $\quad$ constant derived by best-fit relationship between blackbody temperature and emitted spectra radiance

$\mathrm{A}_{\text {pixe:l }} \quad$ ground sampling area

$\mathrm{L}_{\mathrm{p}}$ : $\quad$ atmospherically corrected TIR radiance per pixel $\quad\left[\mathrm{Wm}^{-2} \mathrm{sr}^{-1} \mu \mathrm{m}^{-1}\right]$

$\mathrm{L}_{\mathrm{bg}}$ : atmospherically-corrected mean radiance of neighboring non-fire pixel $\quad\left[\mathrm{Wm}^{-2} \mathrm{sr}^{-1} \mu \mathrm{m}^{-1}\right]$

\section{FIELD INVESTIGATIONS}

On the coal fire zone under investigation consists of three discrete burning zones. Due to the limited time for field measurements it was specified in advance that only a part of a fire zone would be investigated. Owing to the fire characteristics, accessibility and topography a sub area of fire zone 1 on top of a flat hill was selected.

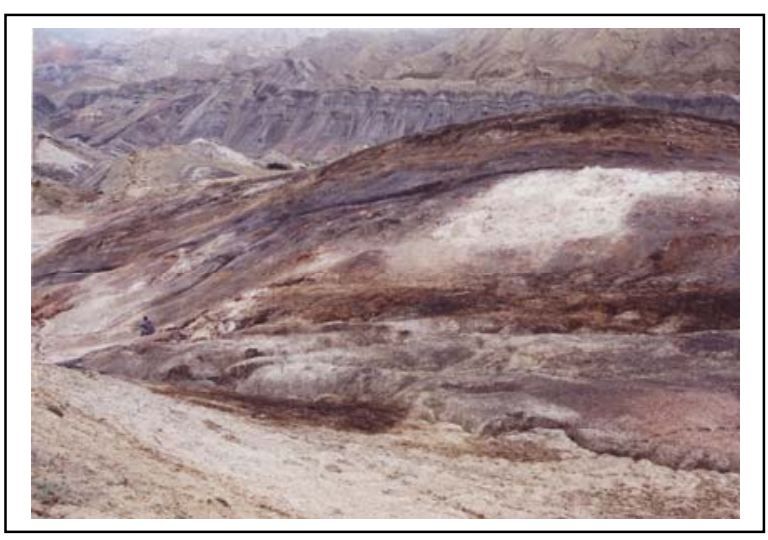

Figure 1: Overview of selected test area

\subsection{Gas measurements}

For the determination of gas emissions different procedures have to be applied depending on temperature, emission site (soil, fractures) and gas flow rates.
For high temperature vent systems the gas flow was determined by a differential pressure measurement. Two circular conduits are placed into one another. The front end of the outer tube is closed and only the inner tube is exposed to the gas stream (stagnation port). At a distance from this opening lateral perforations are penetrating the outer tube only (static ports). When the tube is placed parallel to the flow direction the inner tube is exposed to the gas flow which generates a dynamic pressure, the outer tube is exposed to the ambient pressure. The two conduits are connected to a highly sensitive differential pressure sensor where the pressure difference is measured. According to Bernoulli's Equation the differential pressure depends on the ambient pressure, the gas density and flow velocity.

Closed chamber measurements (a passive method) have been applied. A circular chamber (cylinder), made of steel or temperature resistant Plexiglas, with in- and outlet connectors is placed leak-tight on the soil and the concentration increase (or decrease in case of a gas sink) is measured as a function of time. To ensure a constant homogenization of the gas in the flux chamber a small vane is placed in the chamber or the gas is circulated through the chamber and the measurement device by an internal pump. The concentration of the gas in the chamber is increasing in several phases. At the beginning the concentration build-up is non-linear followed by a linear increase with time. Gas concentration is then asymptotically approaching a maximum value (saturation of the chamber). The gas flow from the soil or vent is determined from the linear increasing concentration:

$$
Q=\frac{d c}{d t} \cdot \frac{V_{C}}{A_{C}}=\frac{d c}{d t} \cdot h_{C}
$$

where:

Q: $\quad$ gas flow

$\mathrm{dc} / \mathrm{dt}$ : concentration increase over time,

hC: $\quad$ height of the chamber

$\mathrm{VC}$; AC: volume and base area of the chamber respectively.

\subsection{Temperature measurements}

For all emission chamber measurements the related temperature was determined by a Multimeter, using a $20 \mathrm{~cm}$ long probe (range $-40^{\circ} \mathrm{C}$ to $400^{\circ} \mathrm{C}$ ) inserted into the soil. The temperature of the gas flow quantified by differential pressure was determined using the build-in temperature sensor in the pitot-tube. 
For a part of the area (approx. $3100 \mathrm{~m}^{2}$ ) a local grid was established using a tape and a local reference point mesh rather than relying on GPS coordinates ( accuracy $5 \mathrm{~m}$ ). Within this area at $\sim 250$ points temperatures at and below surface have been measured.

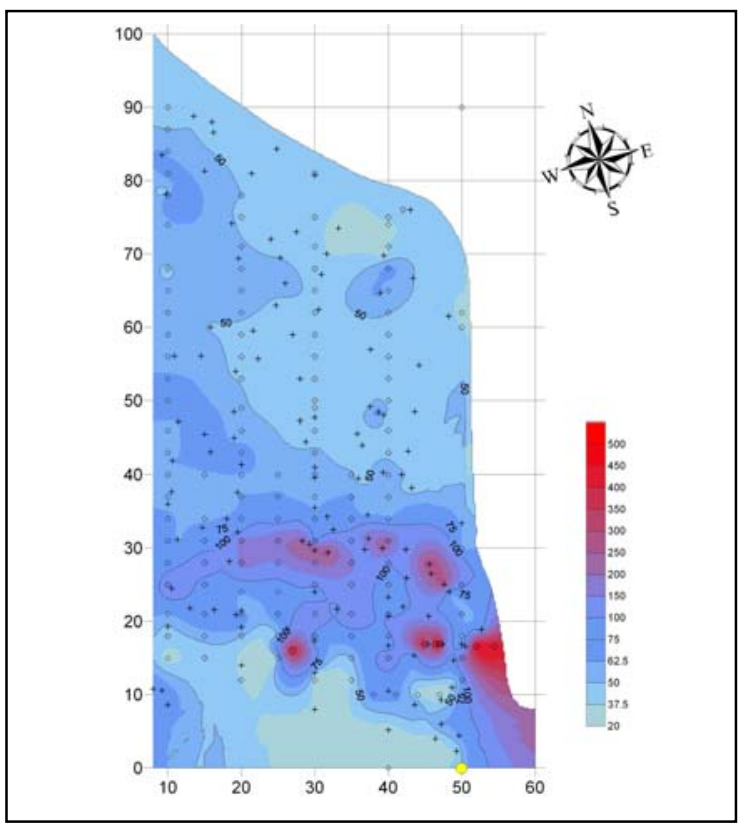

Figure 2: Measured temperatures.

\subsection{Correlation between temperature and gas}

Based on the surface percentage, the average $\mathrm{CO}_{2}$-emissions and the other assumptions, the total $\mathrm{CO}_{2}$-emission in this subzone, can be estimated to $\sim 3400 \mathrm{t} /$ year. Highest normalized flow rates $\left[\mathrm{kg} / \mathrm{year} / \mathrm{m}^{2}\right]$ naturally occur in the fracture system, but also the zone around the outcropping coal seam exhibits large normalized flow rates. Remarkably, the highest total emissions [ $\mathrm{t} / \mathrm{year}$ ] result from the coal seam and the surrounding area $(\sim 30 \%)$. The crack system produces approx. $25 \%$ of the total emissions; the remaining $45 \%$ originate from the surrounding soil covered area. But it hast to be stated clearly, that a direct cross plot of temperature vs. flow rates does not reveal a conclusive correlation.

\subsection{Calculated energy release based on ASTER data}

The area was recorded by ASTER night time images during the first 6 month 2007 at nearly regular times. In total eight images could be analyzed and the corresponding energy release of each scene was calculated for those pixel determined as thermal anomaly. Atmospheric correction was performed, using the ATCOR model [3], based on lookup tables calculated via the MODTRAN code [4].
The average value based on the ASTER night time images was calculated to $0.64 \mathrm{kWm}^{-2}$. The calculations based on field measurements done by Chinese experts result for the complete fire zone summarizes up to $0.81 \mathrm{kWm}^{-2}$ (area: $\left.68.225 \mathrm{~m}^{2}, \Delta \mathrm{T} 282 \mathrm{~K}\right)$.

\section{CONCLUSIONS}

The results obtained so far indicate that:

- the total amount of CO2-emissions for the whole area based on the measurements done in the subarea do not match the calculated emissions,

- The analysis of the multi-temporal ASTER images show promising results, but the preprocessing routines have to be optimized for arid landscapes at comparable high altitudes,

- a better understanding of the energy balance at topographic surface is needed, solar radiation budget and soil heat fluxes have to be taken into account,

- additional investigations have to focus on further investigations how temperature and gas-emission measurements can be coupled to improve further modeling approaches.

\section{REFERENCES}

The authors gratefully acknowledge the support from the Leon Maldonado and his tem from the ASTER project science team at Jet Propulsion Laboratory, Pasadena. The authors also like to thank Mr. Cai from the Fire Fighting Department in Xinjiang and his team for their support and their warmly welcome in Urumqi.

[1] A. Tetzlaff, "Coal Fire Quantification using ASTER, ETM and BIRD Satellite Instrument Data", PhD Thesis, LMU Munich, 2004.

[2] M.J. Wooster, B. Zhukov, and D. Oertel, "Fire radiative energy for quantitative study of biomass burning: derivation from the BIRD experimental satellite and comparison to MODIS fire products", Remote Sensing of Environment, vol. 86, pp. 83-107, 2003.

[3] R. Richter, D. Schlaepfer, Geoathmospheric processing of airborne imaging spectrometer data, part 2: atmospheric and topographic correction", International Journal of Remote Sensing, vol. 23, pp. 2631-2649, 2002.

[4] A. Berk, L.S. Bernstein, and D.C. Rebertson, "MODTRAN: a moderate resolution model for LOWTRAN 7", Final Report GLTR-89-0122, U.S. Air Force Systems Command, USA, 1998. 